\title{
Experimental evidence for the formation mechanism of metallic catalyst-free carbon nanotubes
}

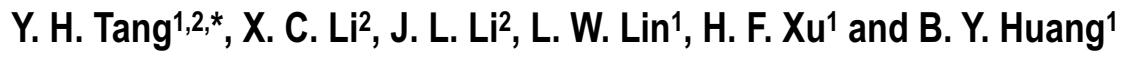 \\ Our work reported that the so-called pure carbon nanotubes (CNTs) can be synthesized without \\ metallic catalyst by chemical vapor deposition (CVD). The as-prepared CNTs have average \\ diameter of $50 \mathrm{~nm}$ and length over several microns. Analysis of intermediate objects in the products \\ indicates that their formation mechanism follows the wire-to-tube model. Besides, according to \\ thermodynamic analysis of the driving force combing with experimental results, we find that the \\ thermal gradient can effectively favor the formation of CNTs in our metallic catalyst-free CVD.
}

Keywords: Carbon nanotubes; Catalyst-free; CVD; Formation mechanism

Citation: Y. H. Tang, X. C. Li, J. L. Li, L. W. Lin, H. F. Xu and B. Y. Huang "Experimental evidence for the formation mechanism of metallic catalyst-free carbon nanotubes", Nano-Micro Lett. 2, 18-21 (2010). doi: 10.5101/nml.v2i1.p18-21

Even carbon nanotubes (CNTs) have been discovered over 18 years [1], the formation mechanism of CNTs is still a puzzled subject. The formation mechanisms have been widely discussed including the vapor-liquid-solid (VLS) model [2-4], the scroll model [5], the lip-lip model [6] and so on [7-11]. The VLS model has been widely accepted for explaining the formation of metallic catalyst-assitsted grown CNTs, in which gaseous carbon is absorbed on the surface of catalyst particles and then diffuses through the catalyst particles, followed by precipitation of carbon with a tubular structure. The scroll model was proposed by Amelinckx et al., in which the curved graphene sheet grows around the existing cylindrical tube and gradually surrounds it, followed by extension growth along the rim of concentric cylinder. The lip-lip model was first proposed by Guo et al. to explain the formation of metallic catalyst-free CNTs by supposing that the lip-lip interaction between shells of multi-wall structure can stabilise the growing edges of CNTs and keeps them open for continued growth, but further researches show that the lip-lip interaction by itself does not stabilizes open-end growth but rather facilitates tube closure $[12,13]$.

The above formation mechanisms focus on a general consensus which the tubular structures of CNTs are formed once the nucleus starting to grow and will sustain during entire growth process. But different from those theorys, our previous work suggested that carbon nanotube could be converted from carbon nanowire upon annealing, according to the near-
edge-X-ray-absorption-fine-structures (NEXAFS) analysis of amorphous carbon nanowire [7]. This kind of formation mechanism (wire-to-tube mechanism) had also been showed by other two groups. Firstly, Yasuda et al. had observed the wire-to-tube evolution by in situ transmission electron microscopy (TEM) observation [14], when they heated a precursor containing polyyne and catalyst salts for growing CNTs in TEM. Secondly, the particle-wire-tube mechanism proposed by $\mathrm{Du}$ et al. [8], similarly involved the wire-to-tube evolution to explain the formation of CNTs, basing on analysis of obtained intermediates and various objects related to CNTs. However, both researches employed metallic calalysts to synthesize CNTs. Thus a question arises whether the wire-to-tube evolution can be realized in the absence of metallic catalysts or not. Recently, we have obtained CNTs in the absence of metallic catalysts with chemical vapor depostion (CVD). This work not only shows our former suggestion [7], but also can obtain the so-called pure CNTs which show really properties of CNTs [15].

The heating furnace with a horizontal tube was employed to grow CNTs. The corundum sheet was used as substrate in order to avoid the influence of metallic particles and was placed at 9 13 $\mathrm{cm}$ away from the center of the furnace tube. After being pumped down to $\sim 10^{-1}$ mbar by a mechanical pump, the furnace tube was refilled with $\mathrm{N}_{2}$ back to the normal pressure. The furnace was then heated to $950^{\circ} \mathrm{C}$ while $\mathrm{N}_{2}$ gas was supplied continuously to maintain the atmospheric pressure 
during the process. As the temperature was stable at $950^{\circ} \mathrm{C}$, the mixed gas of $\mathrm{N}_{2} / \mathrm{CH}_{4}(8: 3)$ was introduced into furnace tube for 1.5 hour. Upon cooling naturally down to room temperature, deposits of black products were found on the substrate. It should be emphazised that no metallic materials or additives was used in the entire progress.

The morphology and microstructure of the products were studied by HRTEM. The analysis of energy dispersive X-ray spectrum(EDS) was also implemented to identify the chemical components of the products. The as-prepared samples analyzed have not gone through any purification processes.

Otherwise, as to clearly understand the synthesis progress, a measurement was carried out to measure the temperature distribution along the furnace tube. When the empty furnace was heated to $950^{\circ} \mathrm{C}$, a pyrometer couple was inserted into the furnace tube to measure the temperatures of the positions from center to outer end of the tube with the dist distance of $1 \mathrm{~cm}$.

From the temperature distribution displayed in the Fig. 1, it is informed that, in the above synthesis progress, the temperature on the substrate $\left(\mathrm{P}_{2}\right.$ position) is $170 \sim 270^{\circ} \mathrm{C}$ lower than the one at the center of the tube ( $\mathrm{P}_{1}$ position), and this temperature gradient is very important factor to gain the CNTs.

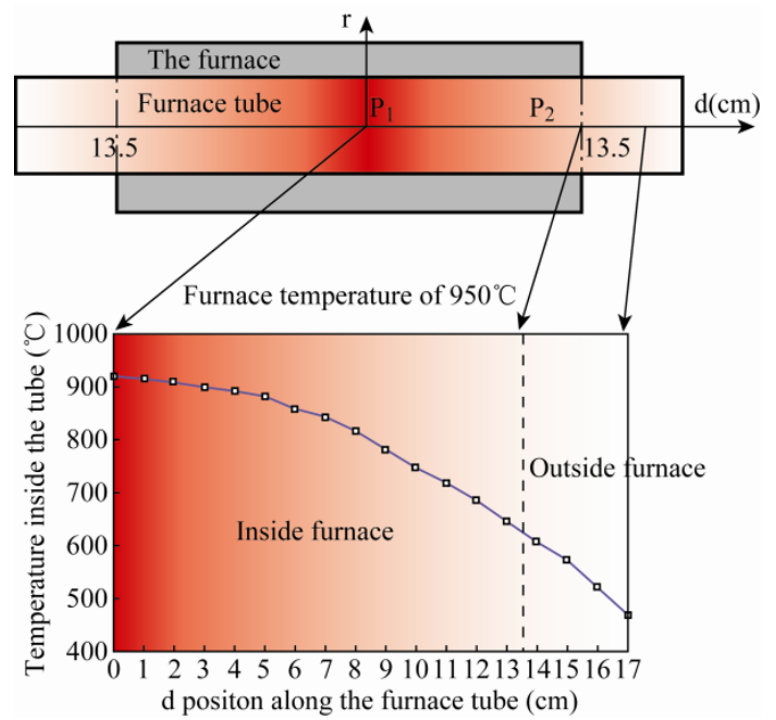

FIG. 1. The temperature distribution along the furnace tube. $\mathrm{r}$ is the radius direction of the tube, $d$ is the position along the tube, $P_{1}$ is the position of center of tube, $\mathrm{P}_{2}$ is the position of the substrate.

The detail explanation is presented in the below article.

In our CVD system, when the furnace was heated to a nominal temperature of $950^{\circ} \mathrm{C}$, the temperature at the center of the furnace tube $\left(\mathrm{P}_{1}\right)$ reached about $920^{\circ} \mathrm{C}$, and the further it was from the central area, the lower the temperature would be. As a result, the temperature on the substrate $\left(\mathrm{P}_{2}\right)$ just reached $650 \sim 750^{\circ} \mathrm{C}$, thus the temperature at $\mathrm{P}_{2}$ is $170 \sim 270^{\circ} \mathrm{C}$ lower than the one at $\mathrm{P}_{1}$.

Figure 2 is a TEM image of the as-products with our method, showing the tubular structure with an inner diameter of approximate $30 \mathrm{~nm}$ and an outer diameter of approximate 50 $\mathrm{nm}$. HRTEM image of the tip with parallel lattices is shown in Fig. 3. The interlayer spacing is $0.33 \mathrm{~nm}$ which is typical for the (002) lattice distance in the ideal graphite, confirming that the as-products are multi-walled carbon nanotubes (MWCNTs).
EDS analysis shows that only carbon spectrum peak can be detected, which is accordance with TEM and HRTEM figures (see Fig. 2 and Fig. 3) that no metallic particles exists in the samples, together with the fact that no metallic materials or additives has been used during the whole synthesis progress, demonstrating that the CNTs are self-assembled without the assistance of metallic catalysts.

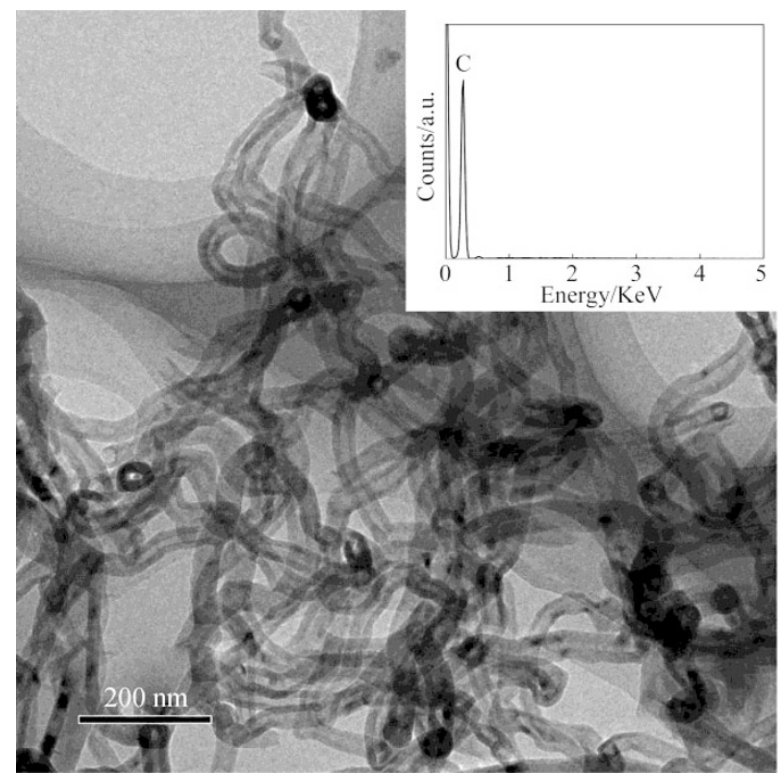

FIG. 2. Low-magnification TEM image of products. Inset is EDS spectrum of products.

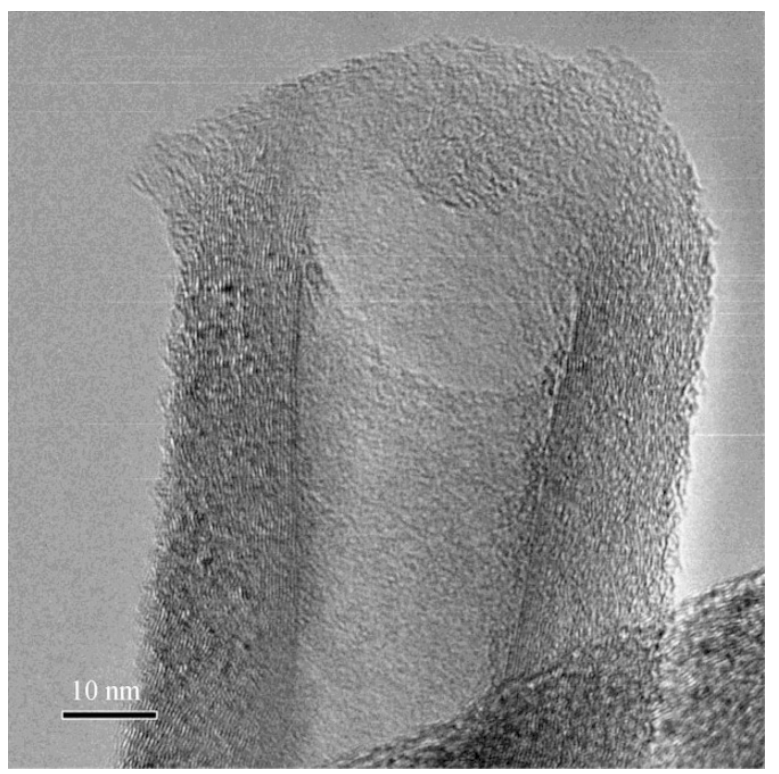

FIG. 3. High-resolution image of the tip of CNT.

In the TEM detection, few samples with special structures as shown in Fig. 4 coexist in this product, sometimes these samples and CNTs with similar diameters may stay together. So, we believe that they are intermediate products which have not completely evolved into CNTs, and they are critical evidences for revealing the formation of CNTs. Basing on the analysis of the samples and CNTs, we proposed that the formation of CNTs in our experiment follows the wire-to-tube model. As schematized in Fig. 5, this model includes three basic steps: (A) 


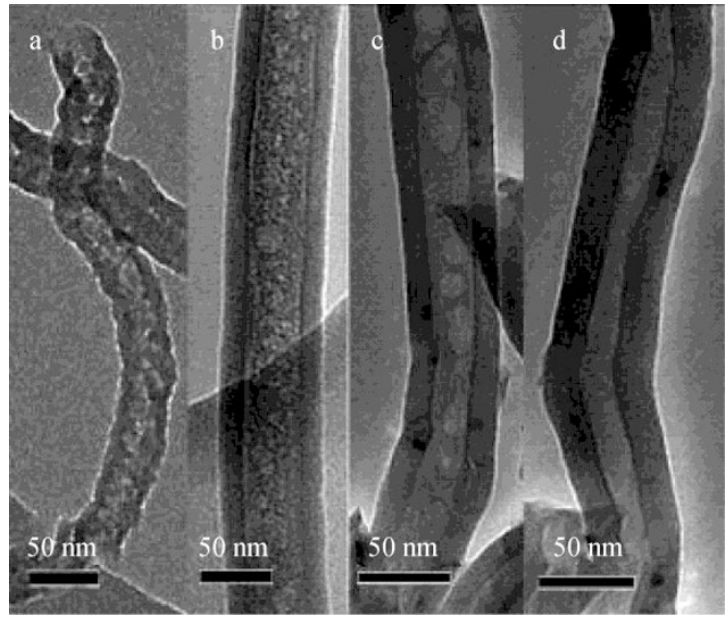

FIG. 4. A set of TEM images showing the evolution progress.

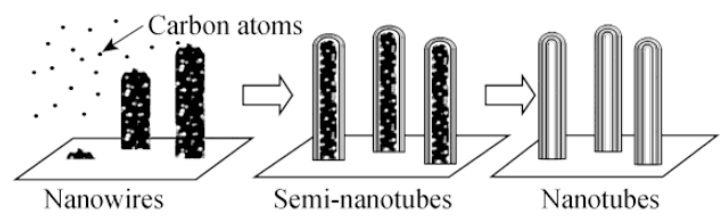

FIG. 5. Scheme of the formation model.

In the first step, gaseous carbon generated from thermolytic $\mathrm{CH}_{4}$ transports and condenses onto the substrate, then forms carbon nanoparticle and grows into carbon nanowire. Whereas there is not conclusive evidence left behind for further understanding the growth of carbon nanowire, here we assume that the generating of defects, such as $\mathrm{sp}^{3}$ carbons, non-hexagonal rings or disconnected bonds, make the carbon nanoparticle come into the one with anisotropy structure, then the following carbon gaseous will sequentially be absorbed at the most stable position on the tip, and keep on growing in one direction for one-dimensional assembly of carbon nanowire. (B) After the first step, the peripheral carbon of the nanowire firstly graphitizes and forms the outter walls, vacating much space for further evolution of inner carbon. Figure 4(b) illustrating a snatch of this scenario that the outter walls were just formed but lots of scraps leaved inside. (C) In the third step, the evolution of inner carbon would become much easier since wide space was left and the former walls were serving as the templates. By the end of this evolution, the hollow structure of CNT was formed. This scenario can be visualized by a set of images of Fig. 4 (b)-(d). Besides, it should be noted that metallic catalysts are dispensable in the whole formation process of CNT.

In the metallic catalyst-free growth process, the driving force for CNTs formation is worth being explored for a further understanding on the growth model. Snoeck et al. proposed that the global driving force for carbon filaments formation is the difference in chemical potential between the gas phase and the carbon filaments [16]. Accordingly, we assume the growth process of CNTs involves a transfer of chemical potential from gaseous carbon to CNTs. Therefore, the driving force of CNTs growth, denoted as $F$, can be expressed as

$$
F=\mu_{c}^{g a s}-\mu_{c}^{C N T s}
$$

Where, $\mu_{c}^{\text {gas }}$ and $\mu_{c}^{C N T S}$ is the chemical potential of gaseous carbon and CNTs, respectively.
In our synthesis, the substrate is placed at $9 \sim 13 \mathrm{~cm}$ away from the center of the furnace tube, and the temperature (deposition temperature $T_{2}$ ) on the substrate is $170 \sim 270^{\circ} \mathrm{C}$ lower than the one (pyrolytic temperature $T_{1}$ ) at the central area, so that a stable thermal gradient will sustain. Additionally, a comparative experiment of the substrate being moved into the central area is carried out but only carbon films can be obtained, indicating the thermal gradient plays a vital role in the formation of CNTs. According to thermodynamic scheme proposed by Hwang et al. [17], the chemical potential of gaseous carbon and CNTs can be expressed by

$$
\mu_{c}^{g a s}=\mu_{c}^{s}+R T_{1} \ln P_{c}^{g a s}
$$

and

$$
\mu_{c}^{C N T S}=\mu_{c}^{s}+R T_{2} \ln P_{c}^{C N T s}
$$

Where, $\mu_{c}^{s}$ is the chemical potential of carbon at the standard state. $R$ is the gas constant. $P_{c}^{\text {gas }}$ and $P_{c}^{C N T s}$ are the partial pressure of gaseous carbon and CNTs, respectively. In the comparative experiment, the pyrolytic temperature $T_{1}$ is equal to the deposition temperature $T_{2}$ which is related to the position of substrate, thus, the $F$ in this situation is expressed as

$F^{\prime}=R T_{1} \ln p_{c}^{g a s} / p_{c}^{C N T s}$

Actually, the $F^{\prime}$ is insufficient for formation of CNTs since no CNTs was obtained in comparative experiment. Whereas, in the case which CNTs can be obtained, $T_{1}$ is higher then $T_{2}$, so the $F$ in this situation will be expressed as

$$
F^{\prime \prime}=R T_{1} \ln p_{c}^{g a s}-R T_{2} \ln p_{c}^{C N T S}
$$

In order to simplify argument, it is assumed that $P_{c}^{\text {gas }}$ and $P_{c}^{C N T S}$ at $T_{1}$ is equal to those at $T_{2}$, thus

$$
F^{\prime \prime}=F^{\prime}+\left(T_{1}-T_{2}\right) R \ln p_{c}^{C N T s}
$$

The value of $\left(T_{1}-T_{2}\right) R \ln p_{c}^{C N T S}$ is validated due to the thermal gradient and consequently enlarges the driving force $F^{\prime \prime}$, which propels the formation of CNTs. We can conclude that the thermal gradient plays a key role in the formation of metallic catalyst-free CNTs.

We obtained the pure CNTs with average diameter of 50 $\mathrm{nm}$ and length over several micrometers with a metallic catalyst-free chemical vapor deposition. Our work offered the experimental surpport for the wire-to-tube model of CNTs which we suggested before. Furthermore, we concluded that the thermal gradient can effectively favor the formation of metallic catalyst-free CNTs, according to thermodynamic analysis of the driving force combining with experimental results.

This research work is supported by the Creative Research Group of National Science Foundation of China (Grant No. 50721003) and the Foundation of the Ministry of Education of China for Returned Scholars (Grant No.2005383). 
Received 19 January 2010; accepted January 28; published online 28 February 2010.

\section{References}

1. S. Iijima, Nature 354, 56 (1991). doi:10.1038/354056a0

2. R. T. K. Baker, M. A. Barber, P. S. Harris, F. S. Feates and R. J. Waite, J. Catal. 26, 51 (1972). doi:10.1016/ 0021-9517(72)90032-2

3. R. T. K. Baker and J. J. Chludzinski, J. Catal. 64, 464 (1980). doi:10.1016/0021-9517(80)90518-7

4. R. T. K. Baker, Carbon 27, 315 (1989). doi:10.1016/ 0008-6223(89)90062-6

5. S. Amelinckx, D. Bernaerts, X. B. Zhang, G. V. Tendeloo and J. V. Landuyt, Science 267, 1334 (1995). doi:10.1126/ science.267.5202.1334

6. T. Guo, P. Nikolaev, A. G. Rinzler, D. Tománek, D. T. Colbert and R. E. Smalley,J. Phys. Chem. 99, 10694 (1995). doi:10.1021/j100027a002

7. S. Iijima, P. M. Ajayan and T. Ichihashi, Phys. Rev. Lett. 69, 3100 (1992). doi:10.1103/PhysRevLett.69.3100

8. M. Endo and H. Kroto, J. Phys. Chem. 96, 6941 (1992). $\underline{\text { doi: } 10.1021 / \mathrm{j} 100196 \mathrm{a} 017}$
9. Y. H. Tang, P. Zhang, P. S. Kim, T. K. Sham,Y. F. Hu and X. H. Sun, et al., Appl. Phys. Lett. 79, 3773 (2001). doi: $10.1063 / 1.1425462$

10. G. Du, S. Feng, J. Zhao, C. Song, S. Bai and Z. Zhu, J. Am. Chem. Soc. 128, 15405 (2006). doi:10.1021/ja064151z

11. S. Amelinckx, X. B. Zhang, D. Bernaerts, X. F. Zhang, V. Ivanov and J. B. Nagy, Science 265, 635 (1994). doi:10.1126/science.265.5172.635

12. Y. K. Kwon, Y. H. Lee, S. G. Kim, P. Jund, D. Tománek and R. E. Smalley, Phys. Rev. Lett. 79, 2065 (1997). doi:10.1103/PhysRevLett.79.2065

13. J. Bernholc, C. Brabec, M. Buongiorno Nardelli, A. Maiti, C. Roland and B. I. Yakobson, Appl. Phys. A 67, 39 (1998). doi:10.1007/s003390050735

14. A. Yasuda, N. Kawase and W. Mizutani, J. Phys. Chem. B 106, 13294 (2002). doi:10.1021/jp0209771

15. Z. Li, L. Wang, Y. Su, Z. Yang, P. Liu and Y. Zhang, Nano-Micro Lett. 1, 9 (2009).

16. J. W. Snoeck, G. F. Froment and M. Fowles, J. Catal. 169, 240 (1997). doi:10.1006/jcat.1997.1634

17. N. M. Hwang and D. Y. Yoon, J. Mater. Sci. Lett. 13, 1437 (1994).doi:10.1007/BF00405056 